\title{
Cavernous Hemangioma of the Renal Hilum Presenting as an Avascularized Solid Mass
}

\author{
Guido Virgilia Savino Mauro Di Stasia Pierluigi Bove ${ }^{a}$ Augusto Orlandi ${ }^{b}$ \\ Paolo Preziosic Giuseppe Vespasiani ${ }^{a}$
}

Departments of a Urology and 'Pathology, University of Rome 'Tor Vergata', and 'Department of Radiology, Casilino Hospital, Rome, Italy

\section{Key Words \\ Hemangioma $\cdot$ Kidney $\cdot$ Benign renal neoplasms}

\begin{abstract}
Hemangiomas are slowly growing benign vascular tumours which rarely involve the kidney. They usually occur in the mucosa or subepithelial tissue of the pelvis, in the pyramid of the kidney and in the cortex. We report a case of cavernous hemangioma presenting as a solid, paradoxically avascular mass of the right renal hilum.

Copyright @ 2003 S. Karger AG, Basel
\end{abstract}

\section{Case Report}

A 32-year-old woman was admitted to the Urology Unit because of intermittent sharp pain in the right flank, associated with vomiting and fever of $>38^{\circ} \mathrm{C}$. No hematuria was reported and the patient had never undergone surgery. The clinical examination was unremarkable. All routine blood tests were normal except for slight increases in the white blood cell count and the erythrocyte sedimentation rate. Urine analysis detected slight hematuria; urine culture was normal. A renal ultrasound scan visualized a roundish, solid, slightly hyperechogenic mass in the right renal hilum. Color Doppler ultrasound did not detect any blood flow in the mass (fig. 1). Urography (IVP) showed a small, round filling defect in the pelvis and upper right calyx with no dilation of the urinary tract. An abdominal CT scan without intravenous contrast medium identified a round mass $26 \mathrm{~mm}$ in diameter with well-delineated edges and regular margins in the right renal sinus. With intravenous contrast medium the lesion was not enhanced (fig. 2). Persistent pain, the mild urinary tract obstruction and our inability to establish the exact nature of the mass made us opt for surgical exploration with the patient understanding that nephrectomy might well be required.

An extrapleural and extraperitoneal thoracoabdominal approach to the right kidney was performed via an oblique incision made over the partially resected tenth rib and extended forward and downward to the anterior abdominal wall. In the renal hilum we found a solid, spherical, reddish-blue mass of about $2-3 \mathrm{~cm}$ in diameter, compressing the renal pelvis and displacing the renal artery and vein anteriorly. Although the mass was strongly adherent to the renal pelvis and parenchyma, it seemed it could be detached from them. The renal pedicle was clamped and dissected. A thin layer of whitish tissue encapsulating the mass was removed and the mass itself was detached from the renal pelvis and parenchyma. During enucleation the bed bled slightly. Bleeding stopped once the mass was detached and did not recur even when the clamp was removed from the renal pedicle. The mass was completely excised and the kidney preserved. Grossly the mass was a hollow nodule, containing a solid, brownish mass like a blood clot, with a thin external capsule.

The postoperative clinical course was uneventful and at present, 18 months after surgery, the patient is perfectly healthy. Several renal ultrasound scans and magnetic resonance imaging 4 months after the operation confirmed the mass was no longer present and that the kidney and urinary tract were normal. Histological findings were indicative of cavernous hemangioma (fig. 3).

\section{KARGER}

Fax +41613061234 E-Mail karger@karger.ch www.karger.com
(C) 2003 S. Karger AG, Basel 0042-1138/03/0713-0325\$19.50/0

Accessible online at: www. karger.com/uin
Dr. Guido Virgili

Cattedra di Urologia, Dipartimento di Biopatologia e Diagnostica per Immagini Università di Roma 'Tor Vergata', Via Montpellier,1

IT-00133 Roma (Italy)

Tel. +390623188341, Fax +3906 238853, E-Mail guidovirgili@tiscalinet.it 
Fig. 1. Color Doppler ultrasound of the right kidney demonstrating a solid, slightly hyperechogenic, avascular mass in the renal hilum.
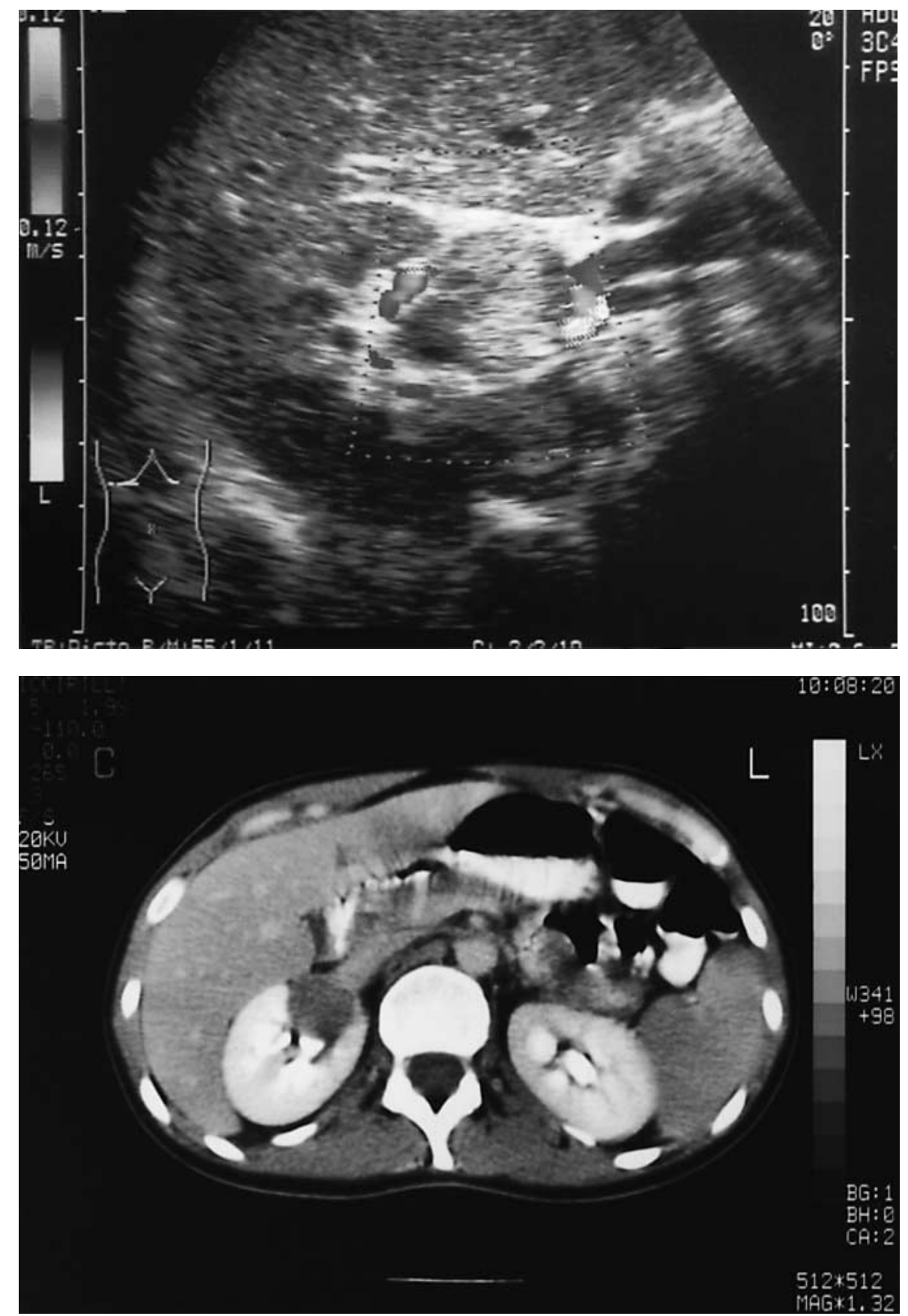

Fig. 2. CT scan with intravenous contrast medium showing in the right renal sinus a nonenhanced round mass $26 \mathrm{~mm}$ in diameter with well-delineated edges and regular margins.

\section{Discussion}

Hemangioma, a benign vascular tumor, probably arises from an embryonic unipotent angioblastic cell residue which does not develop into normal blood vessels [1]. Cavernous hemangiomas are usually found on the skin and mucosal surface but may, on occasion, be observed in the liver, spleen, pancreas and kidneys [2]. Urinary tract involvement is rare and the kidney, followed by the bladder, are most frequently affected in the genitourinary system [1].

About 200 cases of renal hemangioma have been reported since the original description by Virchow in 1867 [3]. Usually renal hemangioma is a solitary and uni- 


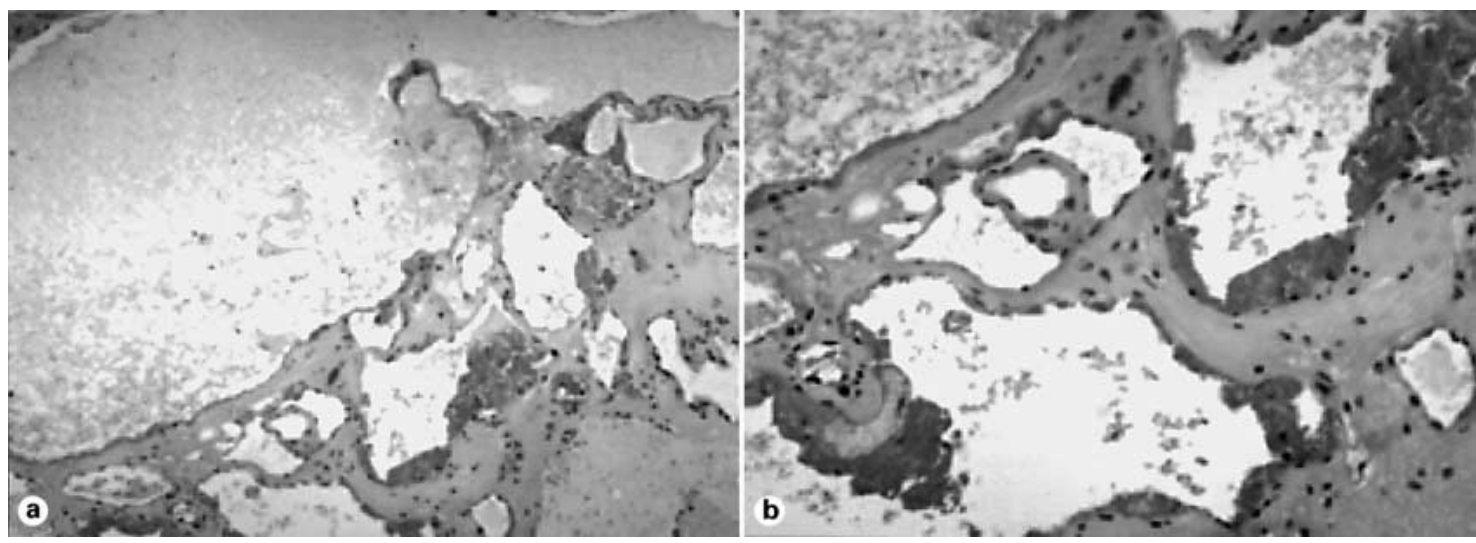

Fig. 3. Histological findings of large dilated blood-filled vessels with a relatively thin wall lined by flattened endothelium. a $\times 125 . \mathbf{b} \times 200$.

lateral mass with no predilection for side or sex and with a peak incidence in patients aged from 30 to 40 years [1, 3, 4]. Multifocal lesions in the same kidney have been reported in $12 \%$ of cases. Hemangioma may be part of a congenital disorder such as the Klippel-Trenaunay syndrome, Sturge-Weber syndrome and systemic angiomatosis $[1,5,6]$. Hemangioma occurs usually in the mucosa or subepithelial tissue of the pelvis $(48.7 \%)$, in the pyramid $(42.1 \%)$ and in the cortex of the kidney $(9.2 \%)[1,7]$. Grossly the soft reddish-blue mass has a diameter of 2 $3 \mathrm{~cm}$. Histologically it is sharply defined, has no capsule and consists of a large cavernous vascular space which may be partially or completely filled with blood and separated by scant connective tissue stroma [2]. Hemangioma can be divided into benign hemangioendotheliomas, capillary hemangioma, cavernous, venous and racemose hemangioma. The cavernous hemangioma is the most common histological type.

When the renal medulla is involved, hemangioma often causes mild deformity of a calyx or hematuria while renal cortex involvement provokes perirenal bleeding and hematoma [2]. Although hemangioma is benign it can cause life-threatening hemorrhage or pyeloureteral junction obstruction. Clinically it is important to differentiate a benign tumor such as hemangioma from a malignancy. Diagnosing hemangioma can be difficult. The IVP may be normal or it may reveal obstruction or transient filling defects due to clots [1]. CT usually shows hemangioma as an enhancing heterogeneous solid mass mimicking a carcinoma [1]. Renal angiography is the diagnostic method of choice but the small size of lesions, the presence of thrombosed vessels and the vascular structure of the tumor can make detection and differentiation from renal carcinoma arduous $[1,8]$. For these reasons, most cases of hemangioma are diagnosed after surgical excision $[4,9]$.

In our patient, the CT scan and the color Doppler ultrasound visualized a nonvascularized mass and diagnosis was oriented towards a hemorrhagic or infected renal cyst, abscess, benign neoplasm and inflammatory mass even though a malignant tumor could not be completely excluded. A hemangioma was not suspected preoperatively because imaging techniques usually reveal it as hypervascularized. Lack of vascularization in a cavernous hemangioma is rare and appears paradoxical. Thrombosis of vessels perfusing the mass may account for its absence in this case. Surgery alone made it possible to see that the mass, although strongly adherent to underlying structures, could be detached from them. The independence of the kidney led to the hypothesis that hemangioma had originated in the retroperitoneal area.

Reports of cavernous hemangioma of retroperitoneal origin are extremely rare [9]. We have found only 1 other case similar to ours but in that instance hypervascularization led to difficulties in differentiating between hemangioma and a malignant retroperitoneal tumor [2]. In our case the diagnostic techniques indicated the mass was benign but could not establish what it was exactly. As it was not malignant we decided not to subject the patient to further tests such as angiography, which would probably have added little to our knowledge. As often occurs in cases of hemangioma, diagnosis was formulated only after surgery. Fortunately, in this patient, features and localization of the mass were such that it could be safely removed and the kidney saved. 


\section{References}

1 Jahn H, Nissen HM: Hemangiomas of the urinary tract: Review of the literature. Br J Urol 1991;68:113-117.

2 Haas Ca, Resnick MI, Abdul-Karim FW: Cavernous hemangioma presenting as a renal hilar mass. J Urol 1998;160:2139-2140.

3 Jahn H, Rasmussen L, Nissen HM: Hemangioma of the kidney. Urol Int 1991;46:200-204.

4 Wallach JB, Sutton AP, Claman M: Hemangioma of the kidney. J Urol 1959;81:515-518.

5 Feczko PJ: Renal hemangioma. Cause of massive hematuria. Urology 1979;13:447-449.

6 Campistol JM, Agusti C, Torras A, Campo E, Abad C, Revert L: Renal hemangioma and renal artery aneurysm in the Klippel-Trenaunay syndrome. J Urol 1988;140:134-136.
7 Weyrauch HM; Berger MM: Hemangioma of the kidney: Report of a case simulating pyeloureteritis cystica. Stanford Med Bull 1951;9: 43-48.

8 Gordon R, Rosenmann E, Barzilay B, Siew F: Correlation of selective angiography and pathology in cavernous hemangioma of the kidney. J Urol 1976;115:608-609.

9 Hull GW, Genega EM, Sogani PC: Intravascular capillary hemangioma presenting as a solid renal mass. J Urol 1999;162:784-785.
Virgili/Di Stasi/Bove/Orlandi/Preziosi/ Vespasiani 